\title{
Augmenting Psychological Restorativeness towards Immersive Design of Underwater Ambiance
}

\author{
Nermin Sena Ozger ${ }^{1, *}$, Gözde Damla Turhan², and Guzden Varinlioglu² \\ ${ }^{1}$ Izmir University of Economics, Department of Visual Communication Design, 35330 Izmir, Turkey \\ ${ }^{2}$ Izmir University of Economics, Department of Architecture, 35330 Izmir, Turkey
}

\begin{abstract}
Discussions of the relationship between humanity and built environment usually focus on the human's relationships with the earth's surface. Less attention has been given to underwater environments, a relatively underexplored research area at the intersection of user experience design and psychology. This paper shows the initial findings of experiments on divers, which can guide the design of augmented reality tools for underwater environment. The comparative evaluation process demonstrates an increase in psychological restorativeness of users in relation to ambiance design, indicating a difference between a specifically designed environment and a naturally vacant area for first time divers. Future studies will explore immersive design of underwater ambiance through augmented reality tools, proposed as a product design and framework, in order to improve psychological restorativeness.
\end{abstract}

\footnotetext{
* Corresponding author: nermin.ozger@iue.edu.tr
} 


\section{Introduction}

During the course of history, humanity has intuitively explored different environments for living. Underwater habitats are environments for which today's technology has potential to extend the ability of humanity to adapt itself to extreme conditions. Although there are various studies, ambiance of underwater architecture and its effects on users are still areas or exploration through alternative methods, due to the complexity involved in such environments. This research focuses on a comparative experiment that measures psychological restorativeness (PR) in relation to underwater ambiance design, and displays a number of findings for furthering the research towards designing a product equipped with augmented reality tools.

The attempts in the literature show that the studies allow users to experience the immersive environment using virtuality tools by recreating a virtual environment in a $3 \mathrm{D}$ platform. However, these completely altered ambiance experiences are considerably different from actual environment itself. In contrast to these findings in the literature, we propose to use the real environment with enhanced its immersiveness through the deployment of 3D artifacts and, an augmented reality (AR) embedded diving mask.

\subsection{Definition of Terms}

Over the last two decades, many studies have shown that restorative environments help replenish psychological well-being, promote recovery from mental fatigue [1], and have beneficial effects on attention restoration [1,2,3]. The interaction between the built environment and psychological restoration was described by Kaplan and Kaplan in 1989 within the context of ART. Attention restoration theory (ART) builds on assumptions about the evolution of human cognitive capabilities in natural environments in relation to the concept of presence. Psychologically, there are three aspects of presence; the user's response to an immersive system; the sense of being there and the extent to which the virtual environment takes precedence over the real one; and finally, the way users refer to their experience as having been to a place [4]. Furthermore, the level of fascination, and stimulation of the cognitive skills are also related to the user's experience, and the ambiance [5]. Therefore, the design of immersive environments can improve the user experience in terms of psychological restorativeness [1].

Facilities that enable visitors to recover from everyday stress, so-called restorative environments, are those where personal adaptive resources and ability to focus are renewed [6]. It is stated that highly populated urban centers create stress on human psychological and physical resources [1]. On the other hand, psychological restoration is supported by natural environments [1,7]; in particular, riversides and seashores have higher levels of impact in comparison with urban spaces, and even green areas [1]. However, in most the cases, the environmental influences of underwater on human psychology are understudied, and therefore, the recreational element is the specific focus of this study.

Diving is a profound physical activity that provides a physically and mentally immersive user experience, and potentially contributes to the improvement of psychological restorativeness. In terms of physical impacts, it can take place in natural reefs, as well as artificial environments created by deploying ships, planes, and other large-scale structures. These wrecks become touristic attractions, but, as seen in the Mediterranean and the Red Sea, artificial reefs can damage the sensitive ecosystems when metal wrecks are inappropriately deployed. 
On the other hand, as an immersive embodied experience, diving mobilizes a complex of human senses that are tactile, audial, odoral, taste, and visual. Even though these senses interact to create material engagement and feeling positionality in space, visual sense is the focus of this paper, since the use of other senses is limited due to water having a denser material characteristic than the air surrounding us. Although visual senses are also distorted by making objects seem 33\% bigger and closer [8], diving masks overcome this problem. The distortion in audio is a greater problem because there is no satisfactory equipment designed to minimize the effects of sound, traveling four times faster underwater than on the surface, making it difficult to detect the direction and the decibel level of the sound. Tactile senses present similar challenges, as water speeds up heat-loss by 25 times compared to air.

To sum up, underwater diving is an activity in which human senses are stimulated and psychological restorativeness is provided by nature, so that human abilities such as learning, paying attention increase. These features are the basis for re-creating an underwater experience with augmented reality tools for divers.

\subsection{History of underwater explorations}

In order to understand the possible scenarios for underwater habitations, it is important to review the pioneers in the field. In the $15^{\text {th }}$ century, legend has it that Alexander the Great ordered a glass barrel to be dropped into the sea. The first records of such efforts in Greece date back to 1538 [9]. These so-called "diving bells" were lowered beneath the sea while the pressure was equalized by creating a vacuum with the air inside. This principle allowed divers to breathe under the surface.

An alternative diving system was not designed until 1828, when John Deane and his brother modified a suit made for firefighters to enter smoke-filled buildings. The new suit consisted of a copper helmet big enough to completely cover a diver's head, attached to a cloth body suit to protect from cold water, and weighted shoes to counteract the positive buoyancy created by the air in the suit. The air was supplied through a pump on the surface, and the helmet was not sealed, so it flooded when the head was turned at an angle. Later, Augustus Seibe developed the diving suit by connecting the helmet via seal to prevent it from flooding [10]. Without the technology to compress air into a container (and on-demand regulator as the fundamental equipment) at this time, it was impossible to develop a selfcontained underwater breathing apparatus (scuba). It was Cousteau, the first scuba diver, who first used independent air supply with the invention of the aqualung regulator, freeing him from the need to be connected to those above the surface.

All of these early attempts led humanity to remain under the sea for longer periods while observing the marine life. In general, the explorations underwater started with attempts to create a space through glass barrels and diving suits, and eventually developed into scuba. The development can be reviewed from spatial designs to product design. Nevertheless, diving equipment has not been sufficiently integrated with digital technologies for the purpose of enhancing the psychological restoration. Therefore, an experiment was conducted to explore an alternate immersive underwater experience. 


\section{Experiment on the relationship between PR and design of immersive underwater ambiance}

It is observed that functioning of daily routines typically demands our capacity to direct attention, and that this capacity may weaken over time, causing direct attention fatigue. This, in turn, results in negative emotions, irritability or decreased sensitivity to interpersonal issues [1]. This depletion of directed attention can be restored by resting in such environments where this mental fatigue can be reduced [7], although the occurrence and intensity of restorative experiences is unpredictable [6].

A literature research revealed three types of restorative impacts on humans: Emotional, physiological, and cognitive impacts evaluated by the psychological restorativeness scales that measure the relationship between diver (user) and underwater ambiance design $[1,5]$, and by the Stroop Effect test to measure the cognitive abilities before and after the activity. According to Pasini et al [11], restorative environments encompass a wide range of settings from wilderness to indoors, and a variety of different scales. In the literature, there are four common identified properties of restorative experience settings: Being away, extent, fascination, and compatibility characteristics of both the imagined/mental domain and the actual/physical domain [5]. When developing the Psychological Restorativeness scale (PRs), two components of a restorative experience were consistently identified as present in each analysis: "being away" and "fascination", and these are the core features of the ART [7].

In relation to these two components of psychological restorativeness scales, recreational diving is defined as a recreational activity to which ART applies, since it provides sense of being away and fascination in terms of both mental and physical domains. At this point, the integration of immersive digital media to designed underwater ambiance can potentially enhance the user experience while decreasing the environmental concerns. Immersive media, such as augmented reality (AR) tools, are being widely explored in relation to user experience. In discussing AR tools, the visual aspect of the interaction with the imposition of three-dimensional computer graphics is often central to discussions; however, any media within a synthetic space, provided by, for instance, sound or haptic features, can increase the users' sense of reality for various explorations [12].

Digitization of the ambiance of the built-up environment has a potential to provide alternative solutions to a wider spread of knowledge and experience. Additionally, the use of immersive reality tools could make the experience more appealing and innovative. Through this research, users will be able to experience a completely innovative underwater ambiance without the harm to the built-up environment caused by current applications.

\subsection{Methodology}

This study starts by proving how psychological restorativeness (PR) is enhanced through an immersive underwater ambiance design. Designed space has been shown to improve PR more compared to the naturally vacant space. Furthermore, it is known that immersive media improves underwater user experience [13]. These results led us to explore alternative of digital applications, i.e. augmented reality (AR) to experience underwater ambiance, while reducing the need for physical and artificial attraction points. For this, an AR embedded diving mask design is envisioned. For acquisition and dissemination of knowledge, augmented reality (AR) are often used for making physical content digitally accessible, especially when the actual content is rather inaccessible. However, in this study, the concern was over issues of physical impacts rather than accessibility, and users' psychological 
responses were studied to gain insights into the outcomes of an immersive underwater experience.

Research on restorative environments shows the precise outcomes for experience of nature, generally by comparing an attractive natural ambiance to the less attractive built environment [14]. In parallel, this study compares the psychological effects of a built underwater ambiance and vacant underwater ambiance with the aim of identifying whether differences in design have a critical role in the level of psychological restorativeness in terms of ambiance experience. Moreover, psychological tests are critical to measuring the level of changes in psychological restorativeness, but these have rarely been conducted within the framework of user experience design, especially for underwater studies.

In order to promote and integrate psychological restorativeness (PR) as one of the criteria in the design of underwater ambiance, the PR and Stroop Effect tests were conducted with 30 divers in shallow waters. PR scales were designed according to studies by Han and Hartig $[1,5]$. The cognitive response durations were measured by the Stroop Effect tests. The initial results have shown that users diving for the first time in an artificially created built environment showed a greater amount of psychological restorativeness than those in a naturally vacant area. Moreover, through The Stroop Effect tests, an increase in participants' cognitive abilities was recorded.

To elaborate, in the Izmir/Karaburun area, August 2018, the corresponding author designed the dive site, deploying clay-made archaeological replicas (i.e. columns and amphoras) at shallow levels (between 5-7 mt depth) on the seafloor, composed of sand, seagrass, and rocks (Fig.1). Variables such as the users' physical features, e.g. weight, were not taken into consideration. The environmental conditions where the tests conducted were pegged. Each trial dive lasted for 20 minutes $(-/+3$ mins $)$, and the average water temperature was $21^{\circ} \mathrm{C}$.

The ambiance design was planned according to the trial dive route underwater. The artifacts were carefully placed in three groups; first group consists of a small group of amphoras as a starting attraction point; second part is a group of amphoras at a deeper level, and third group involves columns, amphoras, and other clay artifacts, as the final stop for the trial divers. 
For the pre- and post-dive, participants were asked to indicate the colors of rectangles, to ensure that they could clearly see the colors. Second, they were asked to read aloud the words that were written in a different color from the word they signified, e.g. the word "red" written in blue. Finally, they were asked to identify the color each word was written in, ignoring the word itself, for instance, the word "red" written in blue, should be identified as blue. The time taken for reading and the number of errors were recorded for each phase. After the trial dive, divers evaluated their experience by the self-rating psychological restorativeness scales.

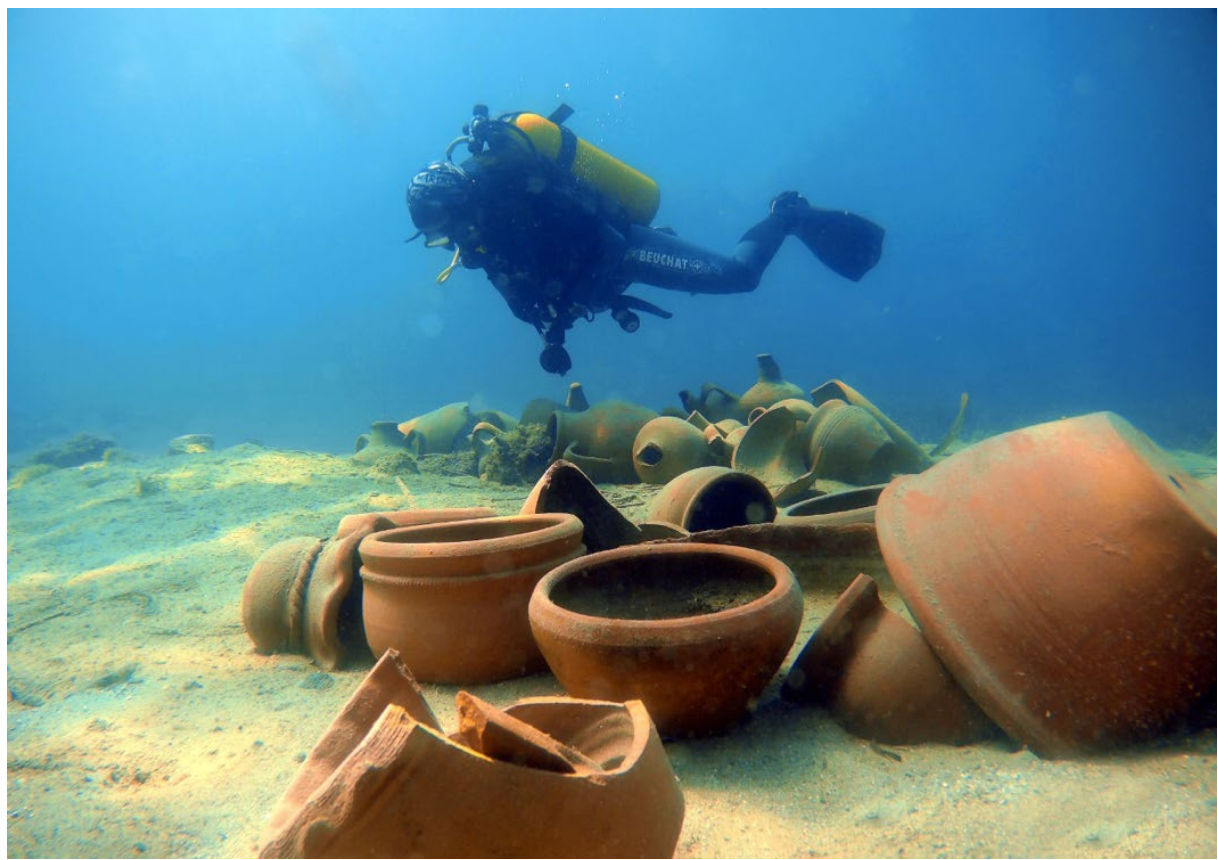

Fig. 1. Deployed columns and amphoras, Cagri Guven (2018).

\subsection{Findings}

The experiment showed a direct relationship between the ambiance design and psychological restorativeness. According to the Stroop Effect test results, Group A, as experiment group, shows greater progress than Group B, as the control group, in all three phases. In indicating the colors of rectangles, the average response time fell by $14 \%$ for Group A, and $8.50 \%$ for Group B after the trial dive. The improvement in recorded time average for reading words is $11.70 \%$ for Group A, and 6\% for Group B. The improvement for indicating the colors of words for Group A is $13.32 \%$, and $10.22 \%$ for Group B.

The self-rating psychological tests were conducted post-dive. They aim to obtain an insight about the divers' psychological and physical reaction to the underwater. The results of the post-dive self-rating psychological tests state that the divers were more likely to be curious for further exploration of underwater, especially when they noticed that the ambiance was specifically designed. Moreover, the test group indicated that they would spend more time underwater to explore the area after they started to be familiarized with their surroundings. This proven point can be also interpreted as the desire to be around artifacts they know of. 
These findings are complementary to the hypothesis that the design has a critical role in the level of psychological restorativeness in terms of underwater ambiance experience. Diving as a physical activity has an effect on the users, however, the ambiance design has a greater impact on the users' psychological restorativeness in terms of providing a challenging immersive experience.

Although artificial environments created by deploying ships, planes, and other large scale structures are attractive to many divers, an integration of augmented reality (AR) technologies can further facilitate this experience, along with a virtual ambiance design. In order to understand the potential for AR to enhance PR within an underwater ambiance design, an AR-embedded mask design is proposed.

\section{Proposal for an immersive underwater ambiance}

For underwater ambiance, having contact with water elements is essential. In this sense, the "immersion" into the water helps us to dissolve categorizations of water. The very essential activities of swimming and diving, as the very basic forms of immersion, allow the body to interact with the environment. Thus, we did not try to duplicate the underwater environment, as the immersion is not easily reproduced and transformed into the virtual world [16].

We consider augmenting the real environment, rather than re-creating a virtual one by adding a visual concept. As indicated before, diving uses all the senses, but our focus has been on the visual effects. Thus a diving mask (Fig.2), which is used for a clear vision underwater, will be designed using augmented reality (AR) technologies. The mask will enable divers to experience the ambiance through $\mathrm{AR}$ and to see both the real environment and the embedded 3D artifacts.

In order to explain the design of a 3D immersive environment, an insight from the existing literature is briefly reviewed. The approaches might vary from non-realistic re-composition of space as in the case of Char Davies' VR work from 1995, to recent mobile apps, i.e. BBC Civilizations. The level of reality/virtuality depends on the equipment and embodiment. In her work entitled Osmose, Davies [17] explored recreation of underwater ambiance from a fictional viewpoint. However, interaction of the user with the virtual environment copies the movements of a diver. The user's movement in virtuality is through her bodily movements, and breathing rhythm allows the user to move up and down. Thus, the ambiance and movements of the underwater environments are recreated.

Another study exemplifies the use of visual graphics and navigation in order to experience the underwater ambiance. The system works through kinesthetic sensors that enable divers to interact with the underwater space through their own motions. Their aim is to recreate the sensations and physical requirements of scuba through design, without intruding into the space [13]. 
In our design proposal, we do not attempt to recreate a virtual underwater environment and movements, but, instead, the ambiance will be augmented through a transparent glass visor/screen, without changing the overall diving experience. The diving mask, a mandatory equipment for the divers, will also have additional info-graphics displayed in the screen while the diver/user experiences the actual underwater environment. We envision AR-embedded diving mask that will allow divers to see the objects through markers, thus improving the underwater space experience and psychological restorativeness.

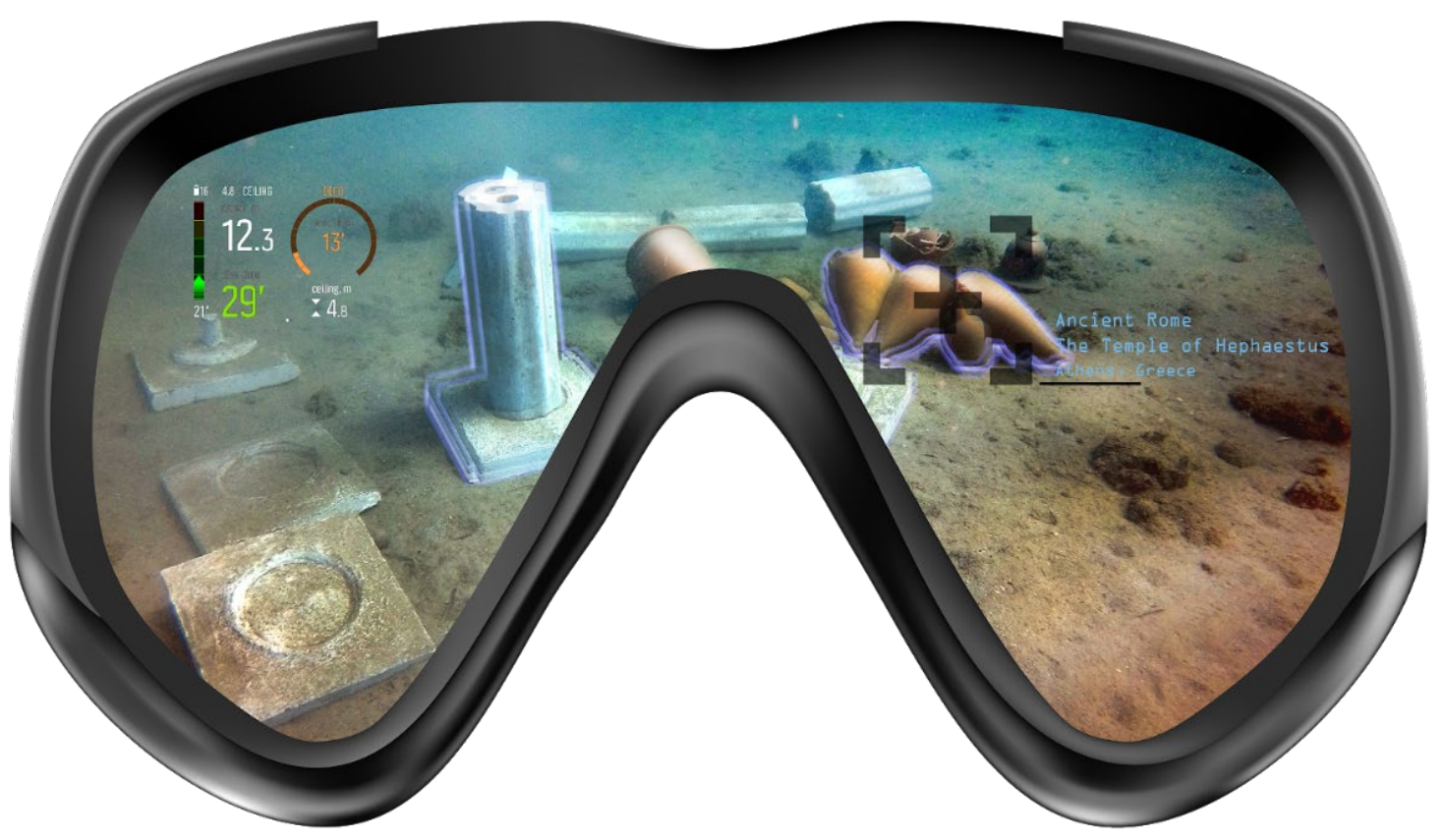

Fig.2 Conceptual AR-embedded diving mask design to experience underwater ambiance showing infographics, by the corresponding author (2018). 


\section{Conclusion and future research}

The genesis of this study is to create a link between ambiance design, and user's psychological restoration from a designerly perspective. Once the relation is established between the ambiance as an experience, and psychology, the inclusion of the innovative technologies of AR will potentially improve the potential for design to play a role in psychological restorativeness. The self-rating psychological restorativeness tests are to measure the improvement, if any, caused by the immersive ambiance experience. The tests are the first step to understanding the relation between three key concepts. User experience, ambiance design, and psychological restorativeness.

During the test phase, the goal was to enhance psychological restorativeness through an immersive underwater ambiance design. From a designerly perspective, the influence of underwater ambiances on the user's psychological restorativeness was measured, taking into account environmental concerns. Regarding underwater ambiance design, the findings showed a direct correlation between the built environment and psychological restorativeness, and cognitive abilities. More research is needed to validate the changes in restorativeness, and to create a greater level of interaction between the user and objects. The immersive characteristics of AR provide attention restoration through providing sense of being away, thus, digital technology can be integrated as a tool to meet the challenge of providing an immersive underwater ambiance.

The Restorativeness scales and Stroop Effect tests proved that the immersive design has a positive impact on user's psychological restorativeness. Additionally, even though the structure of immersive design has been shown to have the power to affect the outcomes, there is a need to study the input of the virtual elements such as AR.

Future studies have the potential to enhance the understanding of immersive ambiance design and the psychological restorativeness. The results of the tests will shape the virtual immersive ambiance design and help to eliminate exaggerated physical interference in the underwater environments. Although AR can never replace reality, it can be integrated or overlapped into the real world experience through design; as such, it has a role to play in improving psychological restorativeness and the quality of the experience through the design of underwater ambiance.

We would like to thank Efes Diving School at Karaburun and the diving instructors Cengiz Arslan, Erkan Ayvazoglu and Burak Bambul for their support in deployments; Taner Yilmaz for supplying the clay artifacts; Asst. Prof. Dr. Burak Erdeniz for his academic contributions in Psychology; Cagri Guven for underwater photography; and the significant part of this research, the participants. 


\section{References}

1. T. Hartig, K. G. Korpela, W. Evans and T. Gärling, A measure of restorative quality in environments, Scandinavian Housing and Planning Research, 14:4 (1997)

2. C.A. Lewis, Gardening as a healing process, in M. Francis, and R.T.J Hester (ed), The Meaning of Gardens: Idea, Place and Action. The MIT Press, Cambridge, pp.244-251 (1990)

3. C.M. Tennessen, B. Cimprich, Views to nature: Effects on attention. J Environ Psychol 15:7785, doi: 10.1016/0272-4944(95)90016-0 (1995)

4. D. Jain, M. Sra, J. Guo, R. Marques, R. W-u, J. Chiu, and C. Schmandt, Immersive Terrestrial Scuba Diving Using Virtual Reality, Proceedings of the 2016 CHI Conference Extended Abstracts on Human Factors in Computing Systems - CHI EA '16 (2016)

5. K.T. Han, A reliable and valid self-rating measure of the restorative quality of natural environments (2003)

6. R. Kaplan, S. Kaplan, R.L. Ryan, With People in Mind: Design and Management of Everyday Nature, Island Press, Washington, DC (1998)

7. S. Kaplan, The restorative benefits of nature: toward an integrative framework. J. Environ. Psychol. 15, 169-182 (1995)

8. https://www2.padi.com/blog/2017/12/20/scuba-diving-makes-smart/

9. F.X. Ross, Undersea Vehicles and Habitats: The Peaceful Uses of the Ocean. New York: Thomas Y. Crowell (1970)

10. https://www.deepblu.com/post/81641b20107c11e7aa9ad181bc785596

11. M. Pasini, R. Berto, M. Brondino, R. Hall, C. Ortner, How to Measure The Restorative Quality of Environments: The PRS-11 (2014)

12. T. Shute. Is it OMG finally for augmented reality? interview with Robert Rice. Ugotrade: Virtual Realities in world 2.0. (2009)

13. Jain, D., Sra, M., Guo, J., Marques, R., Wu, R., Chiu, J., \& Schmandt, C.. Immersive Terrestrial Scuba Diving Using Virtual Reality (pp. 1563-1569). Association for Computing Machinery (ACM). https://doi.org/10.1145/2851581.2892503 (2016)

14. B. Erdeniz, E. Serin, M. Aslı, D. Şenol, and G. Alankuş, Virtual Reality - The Effects of Simulated Virtual Reality Environments on Psychological Restoration (2016) 8

15. T. Shute. Is it OMG finally for augmented reality? interview with Robert Rice. Ugotrade: 
Virtual Realities in world 2.0. (2009)

16. Varinlioglu, G. The FluidExperience of Space: Physical Body in Virtual Spaces Over an Analysis of Osmose.Master Thesis. Bilkent University (2013)

17. Straughan, E. R. (2012). Touched by water: The body in scuba diving. Emotion, Space and Society, 5(1), 19-26.doi:10.1016/j.emospa.2010.10.003 